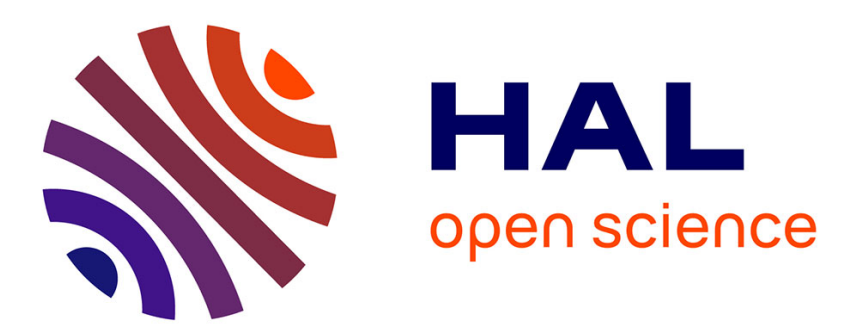

\title{
De l'innovation biomédicale à la pratique de masse : le dépistage prénatal de la trisomie 21 en Angleterre et en France
}

Carine Vassy

\section{- To cite this version: \\ Carine Vassy. De l'innovation biomédicale à la pratique de masse: le dépistage prénatal de la trisomie 21 en Angleterre et en France. Sciences Sociales et Santé, 2011, 29 (3), pp.5-32. 10.3917/sss.293.0005 . hal-00955991}

\section{HAL Id: hal-00955991 \\ https://hal.science/hal-00955991}

Submitted on 5 Mar 2014

HAL is a multi-disciplinary open access archive for the deposit and dissemination of scientific research documents, whether they are published or not. The documents may come from teaching and research institutions in France or abroad, or from public or private research centers.
L'archive ouverte pluridisciplinaire HAL, est destinée au dépôt et à la diffusion de documents scientifiques de niveau recherche, publiés ou non, émanant des établissements d'enseignement et de recherche français ou étrangers, des laboratoires publics ou privés. 


\title{
De l'innovation biomédicale à la pratique de masse : le dépistage prénatal
}

\section{de la trisomie 21 en Angleterre et en France}

\author{
Carine Vassy ${ }^{1}$
}

\section{Résumé :}

L'usage des techniques de diagnostic et de dépistage prénatals de la trisomie 21 s'est largement développé dans les pays industrialisés depuis les années 70. Cela traduit un changement important de normes, en matière d'acceptabilité de l'interruption médicale de grossesse. Cet article a pour but d'étudier les moteurs de la généralisation de ces tests en France, avec une perspective sociohistorique qui met l'accent sur les jeux des acteurs, mais aussi les caractéristiques organisationnelles du système de santé. Pour cela, on fait une comparaison avec l'Angleterre, où ces innovations sont apparues en même temps ou plus tôt, mais où elles ont été, et sont encore,beaucoup moins utilisées.

Mots-clés : dépistage, génétique, innovation, trisomie 21, échographie

La médicalisation des grossesses et des accouchements s'est accélérée depuis le milieu du XXe siècle. Des innovations ont été mises au point pour maîtriser un nombre croissant de situations jugées à risque pour la femme ou le fotus (Oakley, 2003 ; Carricaburu, 2005 ; Jacques, 2007). Les techniques de diagnostic et dépistage prénatals des anomalies fotales constituent une nouvelle étape de cette médicalisation. Une fois détectés, ces problèmes peuvent rarement être guéris. Quand ils sont jugés graves, on propose aux futurs parents

\footnotetext{
${ }^{11}$ Sociologue, Université Paris 13, IRIS, Bobigny, F-93017 France

CNRS, CERMES, Villejuif, F-94801, France

INSERM, IRIS, Bobigny, F-93017 France

EHESS, IRIS, Paris, F-75006, France
} 
l'interruption médicale de la grossesse. Le cas de la trisomie 21, qui est l'anomalie chromosomique la plus fréquente, est exemplaire. Son dépistage est aujourd'hui proposé systématiquement aux femmes enceintes, en France comme dans la plupart des pays industrialisés (ICHBD, 2007). Cette transformation des pratiques depuis les années 1970 traduit un changement important de normes sociales : l'interruption médicale de grossesse est devenue acceptable pour la plupart des professionnels de santé et le reste de la population, alors qu'elle était illégale avant 1975 en France et qu'une partie des médecins y était hostile (Gaudillière, 2001). Comme dans d'autres domaines biomédicaux, on assiste à une « redéfinition de l'exercice légitime de la médecine » (Bateman, 1998).

Comment passe-t-on d'une innovation scientifique à une pratique clinique généralisée ? L'idée d'un déterminisme technique, selon lequel les innovations s'imposent à la société, comme une force extérieure qui engendre des changements sociaux irréversibles, est tombée en désuétude (Vinck, 1995). Les approches actuelles en sciences sociales mettent l'accent sur la capacité d'évolution des objets scientifiques et techniques, la constitution de systèmes sociotechniques et l'importance des régulations. Dans le domaine de la biomédecine comme ailleurs, la légitimation d'une innovation passe par sa régulation, qu'elle soit formelle via l'établissement de dispositifs règlementaires pour encadrer les pratiques, ou informelle par l'élaboration progressive des usagescourants (Baszanger et al, 2000 ; Gaudillière, 2006). Parmi tous les acteurs qui ont contribué à la généralisation du diagnostic et dépistage prénatals de la trisomie $21^{2}$, deux groupes ont joué un rôle clé : les chercheurs et les cliniciens qui ont créé ou importé les innovations, et les pouvoirs publics qui ont apporté un financement pérenne et un cadre juridique à ces nouvelles activités. Pour mieux cerner leur rôle, cet article propose d'en faire une analyse sociologique en terme d'action publique

\footnotetext{
${ }^{2}$ Pour alléger le texte, l'expression « diagnostic et dépistage prénatal » désignera par la suite le diagnostic et dépistage prénatal de la trisomie 21.
} 
(Lascoumes et al, 2007 ; Musselin, 2005). Cette approche présente l'avantage de relativiser la rationalité des interventions publiques, en montrant leurs effets non intentionnels et non anticipés. Or ceux-ci sont nombreux à faire l'objet de critiques dans le domaine du diagnostic et du dépistage prénatals en France. Outre la critique morale de la finalité eugéniste de ces pratiques, débat dans lequel nous ne rentrerons pas ici, deux autres reproches sont faits actuellement, en particulier par des praticiens. Le premier porte sur la qualité insuffisante de l'information donnée aux femmes enceintes à leur entrée dans le dispositif de dépistage. Le second met en avant le trop grand nombre de tests de diagnostic effectués (le caryotype fœtal), qui se traduit par le décès accidentel de fœtus sains suite à l'acte de prélèvement (principalement l'amniocentèse ${ }^{3}$ ). Ces tests se sont multipliés en France dans les années 90, jusqu'à concerner 11 à $12 \%$ des grossesses, niveau qui s'est stabilisé dans les années 2000 (Carles et al., 2002 ; Agence de la Biomédecine, 2007). Cette augmentation du nombre de tests n'a pas été anticipée par les acteurs et fait l'objet de critiques : la Haute Autorité de Santé a déclaré qu'il y avait trop de caryotypes faits en France (HAS, 2007). Des professionnels ont souligné que le nombre de fœtus sains perdus dans des fausses-couches suite au prélèvement de l'amniocentèse est supérieur au nombre de fœus atteints de trisomie 21 diagnostiqués et avortés (Boog, 2003 ; Nisand, 2001 ; Ville 2005). Or cette augmentation ne s'est pas produite partout. En Angleterre par exemple, où les mêmes techniques sont utilisées, ce taux était un peu inférieur à 6\% en 1999 et il est resté stable dans les années qui suivent (Smith-Bindman et al., 2003). Comment expliquer cette différence ?

Cet article propose de retracer l'histoire de la généralisation de l'usage de ces techniques en France et en Angleterre, pour comprendre pourquoi les initiatives similaires prises par les

\footnotetext{
${ }^{3} \mathrm{~L}$ 'amniocentèse est une ponction de liquide amniotique à travers l'abdomen. Une autre technique de prélèvement est parfois utilisée : la biopsie de trophoblaste, aussi appelée prélèvement de villosités choriales, qui est un prélèvement de tissu placentaire par voie abdominale ou vaginale.
} 
chercheurs et les cliniciens pionniers du diagnostic prénatal n'ont pas débouché sur les mêmes usages cliniques à partir des années 1990. Monika Steffen (2005) a montré l'intérêt de la comparaison internationale pour étudier comment les systèmes de santé européens, qui sont tous des formes d'Etat Providence, évoluent de manière différente face à l'apparition de problèmes identiques, comme par exemple le sida. La comparaison internationale permet d'attirer l'attention sur les caractéristiques institutionnelles des systèmes de santé, comme l'organisation des soins, leur mode de financement ou le degré de centralisation des politiques de santé (Vassy et Keller, 2010). Mais elle a été peu utilisée dans les recherches sur l'histoire du diagnostic et du dépistage prénatals, qui portent en général sur un seul pays, comme on le verra dans les références que nous citons plus loin. Seuls deux travaux comportent une comparaison internationale approfondie et ils mettent l'accent sur les caractéristiques organisationnelles des systèmes de santé. La sociologue britannique Margaret Reid (1991) a comparé la diffusion de l'amniocentèse et de l'échographie fotale dans 12 pays européens. Elle note le rôle clé des médecins pionniers, qui défendent l'intérêt des innovations auprès des pouvoirs publics, ainsi que l'importance des modes de financement des systèmes de santé. Pour elle, le caractère décentralisé de la gestion des budgets au Royaume-Uni y est vraisemblablement à l'origine de l'inégale diffusion géographique des innovations. Elle souligne aussi que la plupart des gouvernements européens délèguent la gestion de ces innovations à la profession médicale car ils craignent une réaction négative de leur électorat à des programmes liés à l'avortement. Barbara Stocking (1991) complète cette analyse en y introduisant une comparaison avec la diffusion des appareils lithotripteurs et la pratique des transplantations d'organes en Europe. Comme les techniques de dépistage et diagnostic prénatal ne coûtent pas cher (sauf l'échographe), elles ont pu être utilisées sur des initiatives médicales locales, et les pouvoirs publics ne sont intervenus que dans un second temps, sans toujours en évaluer les effets. Les associations d'usagers n'ont pas joué un grand rôle dans 
cette diffusion en Europe, vraisemblablement car elles étaient divisées sur l'intérêt de ces techniques ${ }^{4}$. L'auteur note aussi qu'au Royaume-Uni, pays où les dépenses publiques de santé sont faibles, le mode de financement du système de santé ralentit la diffusion de toutes les innovations étudiées. Ces remarques sont-elles encore valides, maintenant que les tests se sont largement généralisés et qu'une technique supplémentaire de dépistage est apparue ?

\title{
Méthodologie
}

Cette recherche s'appuie sur l'analyse d'articles médicaux qui exposent des controverses professionnelles sur la qualité des tests de diagnostic et de dépistage prénatals. Elle comprend aussi l'étude de rapports administratifs et de textes juridiques, et de travaux en sciences humaines et sociales sur l'histoire des tests ou leur mise en œuvre. De plus vingt-huit entretiens semi-directifs ont été effectués en France et quinze en Angleterre, de 2002 à 2011, auprès de chercheurs, professionnels de la santé, responsables administratifs et politiques du système de santé et représentants d'associations d'usagers. Les entretiens portaient sur l'histoire de la mise au point et de la généralisation du diagnostic et du dépistage prénatals de la trisomie 21, ainsi que sur les différentes régulations mises en œuvre. Cet article présente les résultats de recherche, en montrant d'abord les similitudes entre l'Angleterre et la France, puis les différences.

\section{Similitudes anglo-françaises}

\author{
Le développement du caryotype
}

\footnotetext{
${ }^{4}$ Le public intervient donc principalement via les femmes qui demandent individuellement des tests à leur médecin, ou qui leur font un procès après la naissance d'un enfant handicapé (Stocking 1991).
} 
En France comme en Angleterre, le caryotype, technique pour analyser les chromosomes présents dans des tissus humains, a été mis au point par des chercheurs en cytogénétique qui travaillaient dans des centres de recherche publics. Comme le montre Hopkins (2006), les entreprises qui créent de nouveaux médicaments ou du matériel médical n'ont joué aucun rôle dans ces développements. Les pionniers de la génétique n'ont pas de motivation commerciale, mais la volonté de mettre un progrès scientifique au service des médecins et de la population. Ils s'appuient sur des recherches menées aux Etats-Unis en 1956, montrant que les humains ont 46 chromosomes, et sur d'autres études détaillant ensuite les anomalies chromosomiques chez des personnes ayant divers syndromes, puis sur la recherche des médecins français Turpin, Gautier et Lejeune montrant en 1958 la présence d'un chromosome supplémentaire chez les personnes atteintes de trisomie 21 (Gaudillière, 2002 ; Hopkins, 2006). A la fin des années 60 , la technique du caryotype est associée à celle de l'amniocentèse, qui consiste à prélever du liquide amniotique à travers l'abdomen de femmes enceintes ${ }^{5}$. Dans ce liquide se trouvent des cellules fœtales, dont on analyse les chromosomes. Les premiers caryotypes fœtaux sont faits à la fin des années 60 aux USA, en Écosse et en Angleterre et au début des années 70 en France (Hopkins 2006 ; Reid, 1991). Un certain nombre de chercheurs qui développent cette activité en Europe vont se former aux États-Unis. A leur retour, ils mettent en place des coopérations pour que travaillent ensemble des obstétriciens qui font les amniocentèses, des cytogénéticiens spécialistes de l'analyse des cellules, et des médecins qui leur adressent les familles qui peuvent bénéficier de ce nouveau service.

La génétique en tant que discipline et profession se construit en grande partie grâce au développement du caryotype dans les deux pays (Hopkins, 2006 ; Pinell 2004a ; Pinell 2004b). Grâce à cet outil, le généticien peut donner un diagnostic avec certitude à un malade,

${ }^{5} \mathrm{~L}$ 'amniocentèse était déjà utilisée dans les années 50 pour analyser les cellules fœetales et détecter des problèmes liés au rhésus ou au sexe du fœtus (Schwartz-Cowan, 2008). 
ou à une femme enceinte, pour certaines pathologies, là où il lui fallait auparavant se contenter de faire, au mieux, des estimations quant à la probabilité de transmission héréditaire. Un nombre croissant de maladies génétiques est progressivement identifié, mais l'on trouve rarement de thérapie. En matière de reproduction, le caryotype fœetal ouvre de nouvelles perspectives d'intervention car il est associé à l'interruption de grossesse, qui est légalisée en 1967 en Angleterre et en 1975 en France. Il permet d'éviter la naissance de personnes dont on ne saurait pas guérir les anomalies. Or le caryotype fœtal concerne potentiellement un nombre de personnes beaucoup plus important que les seules familles qui souffrent de maladies héréditaires : toutes les femmes enceintes sont à risque d'avoir un enfant trisomique, car l'immense majorité des trisomies sont dues à un problème de fusion des cellules sexuelles maternelles et paternelles lors de la fécondation. Seul un très petit nombre de cas sont des formes transmises par l'un des parents, déjà porteur d'une translocation chromosomique, parfois sans le savoir.

Les généticiens ont d'abord proposé le caryotype fotal aux familles qui venaient consulter pour des anomalies chromosomiques chez l'un de leurs membres. Le but était de permettre à des couples de se lancer dans un projet d'enfant au lieu d'y renoncer, en leur donnant la possibilité d'interrompre la grossesse si le fœtus était atteint. Certains généticiens et médecins étaient partisans de limiter le test à ce public limité car le mode de prélèvement entrainait un risque de fausse-couche important, la technique de l'amniocentèse n'étant pas complètement maîtrisée. Mais rapidement le test fut proposé à d'autres catégories de femmes enceintes, comme les femmes âgées de plus de 40 ans. Certaines étaient intéressées car on savait que le risque de trisomie fœtale augmente avec l'âge de la mère. Les débuts de cette activité sont financés sur des fonds publics : des budgets pour la recherche d'abord, quand la technique est à un stade expérimental, puis des fonds de l'assurance maladie en France et du système national de santé en Angleterre. 
Dans les deux pays, l'activité de diagnostic prénatal a été mise en place par des associations professionnelles. En Angleterre, des médecins qui se spécialisent dans la génétique créent en 1970 la Clinical Genetics Society (CGS) (Hopkins, 2006). En France, une association se crée en 1975 sous la présidence du Professeur Frézal, titulaire de la chaire de génétique à l'Hôpital des Enfants Malades à Paris. Cette Association Française pour le Dépistage et la Prévention des Handicaps de l'Enfant (AFDPHE) regroupe principalement des pédiatres et des généticiens, qui se sont d'abord réunis pour lancer des programmes de dépistages néo-natals. La même année est fondée l'Association des Centres d'Études de Biologie Prénatale, qui regroupe des responsables de laboratoires qui font des analyses de cytogénétique. Dans les deux pays, ces associations contribuent à créer des liens entre les acteurs du diagnostic prénatal et à susciter l'intérêt de nouveaux professionnels de la santé. Elles demandent aux responsables du système de santé des financements pour cette activité. Enfin elles définissent des normes professionnelles en matière de laboratoires, de quantité et de qualité des caryotypes à effectuer, de charge de travail acceptable dans chaque centre, et de procédures pour adresser les femmes enceintes.

La puissance de ces associations professionnelles a fait l'objet de commentaires semblables dans les deux pays. En Angleterre, l'historien Michael Hopkins (2006) montre que la CGS illustre de manière exemplaire la manière dont une communauté médico-scientifique modèle la pratique médicale, sans aucun contrôle extérieur. Il critique l'absence d'agence de régulation indépendante et parle de politique de recherche « cachée ». En France, Monika Steffen (1996) affirme que l'AFDPHE représente un modèle d'autorégulation professionnelle. Patrice Pinell (2004a) parle de l'autolégitimation de cette association qui contrôle la mise en œuvre de la politique du diagnostic prénatal. Pourquoi les associations professionnelles des deux côtés de la Manche ont-elles bénéficié de cette autonomie ? Cela présente de nombreux avantages pour les ministères de la santé, qui leur délèguent la responsabilité de surveiller 
l'introduction des innovations biomédicales dans les pratiques, et évitent d'intervenir directement dans une activité controversée car liée à l'avortement, sujet électoralement sensible (Reid, 1991 ; Hopkins 2006). On peut supposer aussi que cela leur permet d'éviter de faire des dépenses pour mettre en place une instance de contrôle administratif. Ils ont peutêtre été aussi influencés par les travaux d'économistes et d'épidémiologistes qui montrent dans les deux pays que la mise en place d'une politique de diagnostic prénatal est avantageuse pour les finances publiques (Gardent, 1983 ; Farrant, 1985 ; Hagard \& Carter, 1976) : le coût des consultations et des actes de diagnostic prénatal est comparé avec celui de la prise en charge des personnes atteintes de trisomie 21 dans des institutions spécialisées pendant la durée moyenne de leur existence. Le premier est bien inférieur au second.

\section{Similitudes dans le développement des dépistages}

Dans les deux pays, de nouvelles méthodes pour rechercher les foetus atteints de trisomie 21 apparaissent après la diffusion du test de diagnostic. Il s'agit de l'échographie fœale développée dans les années 70, et de l'analyse d'hormones dans le sang maternel, dites marqueurs sériques, à partir des années 80 . Ce sont des techniques de dépistage, et non de diagnostic. Elles ne donnent pas un résultat certain quant à la présence d'une trisomie 21, mais elles en indiquent seulement une probabilité, qui s'exprime sous forme de pourcentage, par exemple une chance sur cent. Elles sont moins coûteuses que les tests diagnostiques et moins risquées car elles ne créent pas de fausses-couches. Elles peuvent donc être proposées à l'ensemble des femmes enceintes pour repérer celles qui sont à risque plus élevé que la moyenne, en utilisant des critères plus fins que le seul âge maternel. Elles sont complémentaires des tests diagnostiques : quand une femme enceinte a été dépistée comme étant à risque élevé, on lui conseille de faire un test de diagnostic pour infirmer ou confirmer 
ce résultat. Il y a eu beaucoup de débats dans les revues médicales internationales pour savoir quel pourcentage retenir comme seuil à partir duquel le risque doit être considéré comme élevé. Ces controverses sont caractéristiques du développement de la biomédecine (Gaudillière, 2006).

L'usage de ces techniques se généralise dans la décennie 90 et conduit à un « irrésistible élargissement des populations » concernées par le diagnostic prénatal (Julian-Reynier \& Bourret, 2006). Dans les deux pays, un nombre croissant de praticiens proposent ces tests de dépistage aux femmes enceintes, tandis qu'ils continuent souvent à prescrire directement un test diagnostique aux femmes considérées comme les plus à risque, en particulier celles qui sont les plus âgées. Peu à peu les techniques de dépistage s'affinent et cette évolution est toujours en cours : les professionnels de la santé cherchent à réduire les erreurs en améliorant l'interprétation des images échographiques et en mesurant des marqueurs qui ont une plus forte corrélation statistique avec la présence d'un fœtus atteint. Il s'agit de réduire le nombre de fotus faussement identifiés comme à risque élevé - faux positifs - ou faussement identifiés comme à risque faible - faux négatifs. A la fin des années 90, des chercheurs combinent les deux dépistages, en développant des logiciels pour calculer un risque unique à partir des valeurs mesurées lors de l'échographie et de l'analyse biochimique. On fait aussi le dépistage plus tôt au cours de la grossesse, au premier trimestre, pour limiter les inconvénients des interruptions de grossesse tardives.

Similitudes dans l'absence d'anticipation des problèmes d'information des femmes

Dans les deux pays, la généralisation des tests de diagnostic et de dépistage est justifiée par des arguments centrés sur les droits des parents : passer ces tests permet aux femmes 
enceintes de savoir si leur fœtus est atteint de trisomie 21. Elles peuvent alors choisir d'avorter, ou de continuer la grossesse en se préparant psychologiquement à la naissance d'un enfant handicapé. Chaque femme doit décider de faire ou non les tests, après en avoir discuté avec le professionnel de la santé qui suit sa grossesse. Celui-ci doit lui proposer ces tests, car sinon il lui fait perdre l'opportunité de recourir à l'interruption de grossesse. Il doit l'informer d'une manière compréhensible et neutre, sans imposer son opinion personnelle sur l'utilité du test.

Cet argumentaire a été élaboré par les généticiens qui ont lancé le caryotype fotal, puis repris par les professionnels de la santé qui s'occupent des femmes enceintes. C'est aussi le discours des pouvoirs publics. Aujourd'hui certains points en sont contestés par des médecins, des associations d'usagers et des spécialistes de sciences humaines et sociales de manière similaire des deux côtés de la Manche.

Dans les deux pays, les professionnels qui suivent les grossesses ont des connaissances parfois insuffisantes sur les tests de dépistage et de diagnostic prénatals (Ville, 2005 ; Favre 2007 ; Marteau 1995 ; Smith et al. 1994a). Certains ont des difficultés à expliquer aux femmes enceintes en quoi consistent ces tests car il faut utiliser des notions biologiques et statistiques complexes. Parfois ils sont mal à l'aise avec le test des marqueurs sériques, qu'ils trouvent peu fiable et anxiogène car sa valeur prédictive positive est beaucoup plus faible que d'autres tests utilisés en médecine : pour cent femmes qui reçoivent un résultat de risque élevé au test du $2^{\mathrm{e}}$ trimestre (avec un seuil de risque à $1 / 250$ ), seule une a un fotus trisomique (Green, 1994 ; Flori \& Gofette, 2006). A ces difficultés s’ajoutent des contraintes temporelles qui gênent la communication dans la consultation. Le praticien doit accomplir de multiples actes pendant une durée limitée : recueillir des informations sur l'histoire médicale de la femme et ses antécédents familiaux, faire un éventuel examen clinique et prescrire plusieurs 
autres examens. Il n'a pas toujours le temps et la disponibilité d'esprit nécessaires pour donner une information claire sur le dépistage prénatal. Or moins le praticien communique d'informations, moins la femme enceinte comprend en quoi consistent les tests et quels sont les choix à faire (Marteau 1992 ; Favre, 2007). Dans ce contexte, certains praticiens proposent les tests de dépistage d'une manière brève et directive, attendant des femmes qu'elles se conforment aux procédures de routine (Williams et al. 2002 ; Mattéi, 2000).

Chez les femmes enceintes, on note parfois une forte anxiété lorsqu'elles sont averties qu'elles sont à risque plus élevé que la moyenne ou quand elles attendent les résultats du caryotype, et cette angoisse ne s'atténue pas toujours avec le retour de résultats rassurants (David \& Gosme Séguret, 1996 ; Green et al. 2004). Interrogées après avoir passé les tests, certaines femmes sont mécontentes de l'information qui leur a été donnée (Seror et al, 2001 ; Statham \& Green, 1993). D’autres ne savent pas quels tests elles ont passé ou quelle maladie ces tests dépistent (De Vigan et al., 2002 ; Statham \& Green, 1993). Leur connaissance du test des marqueurs sériques, et en particulier de l'existence de faux positifs et de faux négatifs, est parfois très limitée (Gekas et al., 1999 ; Smith et al., 1994). Elles peuvent aussi avoir beaucoup de difficultés à comprendre les implications de l'échographie (Favre, 2007 ; Green \& Statham, 1996). Le fait que les tests soient proposés en routine à tout le monde diminue la possibilité pour les femmes de prendre conscience qu'elles ont un choix à faire (ChampenoisRousseau, 2003 ; Green \& Statham, 1996). Certaines femmes ont l'impression d'être rentrées, sans avoir été clairement informées, dans un système dont elles ignorent les tenants et les aboutissants, une sorte d' « engrenage »(Clarke, 1997, Mattéi, 2000). Certaines vont jusqu'à croire que les tests de dépistage de la trisomie 21 sont obligatoires (Favre, 2004 ; Gekas et al., 1999). Les femmes issues des milieux défavorisés ont plus de difficultés de compréhension que les autres (Green et al., 2004 ; Khoshnood et al., 2006). Certaines de ces critiques ont été reprises par des associations d'usagers du système de santé (ORUP, 2004 ; Robinson, 1997). 
Comment expliquer ces contradictions entre ce qu'il était prévu de faire et la situation actuelle ? Comme l'a souligné un généticien britannique, l'extension du dépistage à l'ensemble de la population s'est accompagnée d'effets pervers non anticipés (Clarke, 1997). Les pionniers ont cru pouvoir transposer à l'ensemble de la population le modèle de la consultation de génétique proposée aux premiers utilisateurs. Dans ces consultations, les généticiens recevaient des personnes qui étaient sensibilisées au problème de la naissance d'un enfant handicapé par leur expérience personnelle. Elles avaient fait la démarche de venir consulter et étaient demandeuses d'informations sur l'impact de leur hérédité. Elles étaient reçues par des spécialistes, qui connaissaient bien le sujet et leur offraient une prise en charge individualisée, en prenant le temps nécessaire. Ce généticien contraste le «luxe relatif des consultations de conseil génétique » avec les fortes contraintes temporelles qui pèsent sur les consultations de suivi de grossesse (Clarke, 1997). On peut aussi faire l'hypothèse que le public des premières consultations de génétique prénatale provenait de classes moyennes ou aisées, ayant une plus grande familiarité avec le discours médical que le public varié du dépistage de masse.

La généralisation du dépistage prénatal présente donc de nombreux points communs dans les deux pays, y compris dans ses effets non anticipés, commela difficulté de former les praticiens et la mauvaise qualité de l'information des femmes enceintes. En revanche une autre critique, celle du nombre excessif de tests diagnostics, est essentiellement portée en France. Le graphique 1 montre que dans les années 1990 la France a dépassé fortement l'Angleterre. Comment l'expliquer?

\section{Spécificités nationales}

Graphique 1. Nombre de caryotypes fœtaux rapporté au nombre des naissances en Angleterre

- Pays de Galles et en France par année 


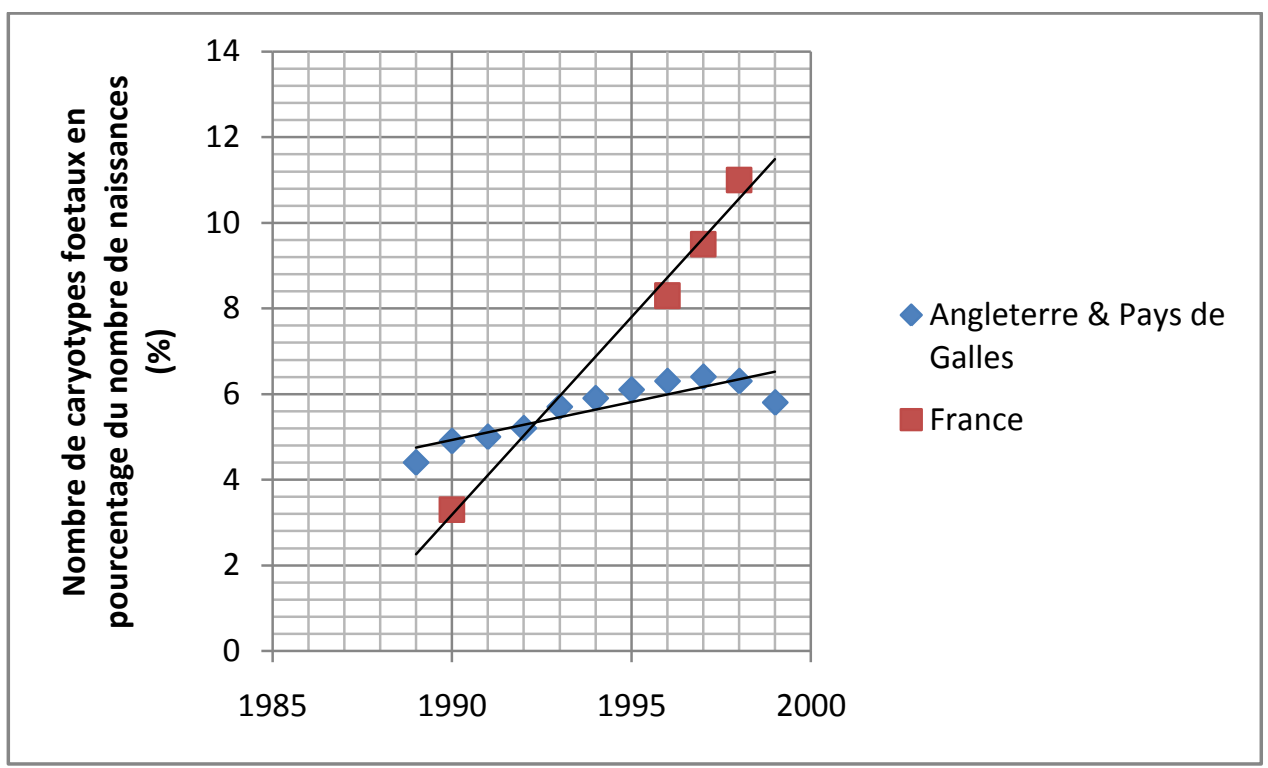

L'organisation et le financement du diagnostic

En France, les pionniers du diagnostic prénatal, rassemblés dans l'association AFDPHE, sont parvenus à fournir ce service gratuitement à l'ensemble des femmes enceintes qu'ils jugeaient à risque, quel que soit leur lieu d'habitation. Pour cela, ils ont négocié le financement des tests avec un petit nombre d'instances parisiennes : la Direction Générale de la Santé et la Caisse Nationale de l'Assurance Maladie (CNAM). Avec ces fonds, l'association finançait les laboratoires de cytogénétique sur l'ensemble du territoire, la plupart étant créés à cette période dans des hôpitaux universitaires (Bourret \& Huard, 1990). Au fur et à mesure que les infrastructures et les compétences se développaient, l'association négociait une extension du nombre de femmes qui avaient accès au caryotype fœtal. Elle a obtenu une première convention avec la CNAM en 1977 pour financer les caryotypes pour les femmes de 40 ans, ainsi que pour les couples ayant eu un enfant porteur d'une anomalie chromosomique ou ayant eux-mêmes une anomalie chromosomique. En 1980 elle a obtenu une nouvelle convention qui a abaissé le seuil d'âge des femmes à 38 ans. En 1988, elle a négocié une autre convention pour financer les caryotypes pour les femmes dont l'échographie fotale avait détecté une anomalie, quel que soit leur âge. En 1991, devant l'augmentation des dépenses, 
1'Assurance Maladie a décidé de financer directement les tests, sans passer par l'association, en les inscrivant à la nomenclature des actes médicaux (Seror, 2003). A chaque étape, les décisions de financement se sont appliquées immédiatement sur tout le territoire national, comme c'est toujours le cas en France. Le rôle symbolique du financement par des instances nationales, que ce soit une association professionnelle ou l'assurance maladie, a augmenté la légitimité des tests auprès des professionnels de santé de première ligne comme auprès des femmes enceintes.

En Angleterre au contraire, des inégalités géographiques d'accès au diagnostic prénatal ont perduré car les négociations pour financer les caryotypes se sont déroulées au niveau local. La quasi totalité des grossesses est suivie par les sages-femmes et les obstétriciens du service public de santé (National Health Service NHS) dans les hôpitaux ou des centres de santé ambulatoires. Il n’y a pas de médecine libérale à la française et le secteur de santé privé, globalement peu développé, s'est peu engagé dans les activités d'obstétrique ${ }^{6}$. Les premiers promoteurs des tests de diagnostic, puis les professionnels chargés du dépistage, ont négocié des financements avec les équipes de direction de leurs hôpitaux, qui elles-mêmes négocient avec les administrations locales de la santé, qui obtiennent leur budget de l'administration nationale. Dans chaque établissement, les priorités en matière d'affectation des budgets sont susceptibles d'être rediscutées en fonction de choix et de rapports de pouvoir locaux. Les programmes de dépistage prénatal ne sont pas toujours jugés prioritaires. Ceci a entraîné une grande disparité de situations en matière de programmes de diagnostic de la trisomie 21, dénoncée régulièrement par les professionnels de la santé comme une «loterie du code postal». Dans les années 80 et 90 , en fonction du lieu où elle habite, la femme enceinte se voit proposer plus ou moins de tests, ou des tests différents, voire aucun test si elle est considérée

${ }^{6}$ En 1994 et 1995, le pourcentage de caryotypes fotaux effectués en Angleterre en dehors du système de santé public (NHS) est de moins de 1\% (Lowther \& Whittle, 1997) 
comme à faible risque du fait de son jeune âge. En Angleterre et au Pays de Galles, le pourcentage de femmes enceintes qui ont un test diagnostique passe de $4.4 \%$ en 1989 à $6.4 \%$ en 1997, puis il décroît légèrement à 5.8\% en 1999 (Smith-Bindman et al., 2003). Et pourtant les tests de dépistage sont de plus en plus utilisés dans les années 90, ce qui pourrait contribuer à augmenter la prescription de tests diagnostiques. En 1989, la principale indication pour faire un caryotype fœtal était l'âge maternel élevé dans $77 \%$ des circonscriptions et en 1999 dans 19\% seulement. Il y a donc eu augmentation des prescriptions de caryotypes suite à des résultats de dépistage biochimiques ou échographiques, mais le nombre de caryotypes n'a pas beaucoup augmenté (Smith-Bindman et al., 2003). Comment s'est donc passée la diffusion des tests de dépistage ?

\section{L'organisation et le financement du dépistage échographique}

La différence entre les deux pays est aussi frappante en matière d'échographie fotale. En France, les premières ont eu lieu dans la seconde moitié des années 70 et elles ont été rapidement financées par la caisse nationale de l'assurance maladie. Les premiers

ultrasonographistes ont négocié le tarif de diverses échographies, dont l'échographie fœtale. A l'époque, celle-ci était utilisée pour obtenir un nombre restreint d'informations : nombre de fœtus, âge gestationnel, voir si le fœtus est vivant et s'il a des anomalies majeures et létales, comme l'anencéphalie (absence totale ou partielle de cerveau). L'offre d'échographies s'est développée au gré des initiatives locales. Ce sont des praticiens du secteur libéral, des gynécologues, des obstétriciens, des radiologues, ou des médecins généralistes, qui prennent individuellement l'initiative de se former aux nouvelles techniques et d'investir dans le matériel, malgré son coût élevé et la rapidité d'obsolescence des machines. En milieu hospitalier, des médecins cherchent à convaincre des directeurs d'établissement et leur tutelle administrative de développer cette activité, en créant des postes et en achetant ces nouveaux 
équipements, mais ils se heurtent aux contraintes budgétaires. L'offre d'échographie foetale se développe plus vite en secteur libéral (Fellous, 1988). A la fin des années 90, environ $38 \%$ des naissances ont lieu en secteur libéral dans des établissements privés, mais les praticiens du secteur libéral effectuent approximativement $60 \%$ des échographies fœtales (Sureau et Henrion, 2005). Le nombre d'examens pratiqués augmente progressivement, en même temps que l'amélioration des performances des appareils. L'échographie devient essentielle à la pratique obstétricale car elle donne beaucoup d'informations sur le fotus, comme par exemple le retard de croissance. Avec plus ou moins de succès, elle peut aussi détecter des malformations diverses, dont la trisomie 21. En 1987, 1'association professionnelle des obstétriciens recommande de faire deux échographies par grossesse. En 1988, la présence d'anomalies lors de l'échographie est reconnue comme une indication justifiant le financement d'un caryotype fotal par l'Assurance Maladie (cf. supra), même si toutes les images produites n'étaient pas de bonne qualité ${ }^{7}$.

Malgré la décision des responsables de l'Assurance Maladie de ne pas revaloriser le tarif de l'échographie fotale en 1994, le nombre d'examens continue d'augmenter. En 1995, l'enquête nationale périnatale montre que $90 \%$ des femmes enceintes ont eu trois échographies ou plus (Blondel et al, 2000). La recherche de la trisomie 21 par mesure de l'épaisseur de la nuque fœtale au premier trimestre commence à être une pratique répandue à partir de 1996. C'est un signe plus fiable que ceux qui étaient utilisés jusque là, ce qui augmente encore l'intérêt pour l'échographie. Selon l'enquête nationale périnatale de 1998, le nombre moyen d'échographies par grossesse est de 4,3 (Blondel et al, 2000). La nouvelle Agence Nationale d'Accréditation et d'Évaluation des Établissements de Santé recommande

\footnotetext{
${ }^{7}$ D'après V. Seror (2003), les pouvoirs publics ont manqué à cette occasion la possibilité d'imposer un contrôle de la qualité des échographies, qui varie beaucoup selon les équipements, les compétences des opérateurs et le temps qu'ils consacrent à effectuer l'examen. A cette époque beaucoup d'échographistes sont des autodidactes et la formation médicale continue ne devient obligatoire qu'en 1996.
} 
en 1998 de faire seulement deux échographies par grossesse. Elle demande la mise en place d'un contrôle de qualité et s'émeut que «les progrès techniques ont précédé les preuves scientifiques et la réflexion éthique » (ANAES, 1998). Mais suite au procès Perruche ${ }^{8}$, un comitécréé sous l'égide du Ministère de la Santé, le Comité National Technique de 1'Echographie de Dépistage Prénatal, qui rassemble l'élite professionnelle des échographistes, recommande de faire trois échographies par grossesse, en faisant la liste détaillée des caractéristiques à vérifier chez le fotus à chaque trimestre (Sureau et Henrion, 2005). Il demande de mettre systématiquement dans le compte-rendu d'examen du premier trimestre la mesure de la nuque fotale, mais de ne communiquer le résultat du calcul de risque à la femme enceinte que si elle le souhaite. A partir des années 2000, le nombre de praticiens qui font des échographies fœtales diminue, à cause de l'absence de revalorisation du prix de l'acte par 1'Assurance Maladie et des procès faits par des parents à des médecins qui n'ont pas détecté des anomalies chez des fœtus (Dommergues et Bessis, 2003). Mais le nombre moyen d'échographies par grossesse demeure supérieur à 4 depuis la fin des années 90, ce qui engendre d'autant plus de prescriptions de caryotypes pour confirmer ou non les résultats.

En Angleterre, la diffusion des examens échographiques est plus lente. En 1991, le Collège Royal des Obstétriciens et Gynécologues recommande de faire un dépistage des anomalies fœtales par échographie pour toutes les femmes enceintes au $2^{\mathrm{e}}$ trimestre (Chitty, 1995). Cette pratique se généralise progressivement, mais il existe de nombreux freins. Certains obstétriciens sont plutôt réticents devant les mauvais résultats de l'échographie à ses débuts, comme la recherche des signes dits mineurs de trisomie 21 , qui amènent souvent à faire des erreurs de pronostic. D’autres observateurs s'inquiètent des effets potentiellement négatifs de l’exposition répétée des fœtus aux ultrasons (Chitty, 1995 ; Oakley, 1993). Un autre obstacle

\footnotetext{
${ }^{8}$ Les époux Perruche entament une procédure judiciaire à partir de 1989 contre des praticiens qui n'ont pas détecté la rubéole de leur fœtus, ce qui a conduit à la naissance d'un enfant gravement handicapé.
} 
est d'ordre économique. Les décisions d'investissement dans le matériel échographique sont prises au niveau de chaque établissement de santé, l'immense majorité d'entre eux appartenant au secteur public. Ils doivent arbitrer entre différentes priorités pour leurs investissements en équipement. Certains s'interrogent sur l'importance des coûts en matériel, mais aussi sur la difficulté de former du personnel qualifié dans le National Health Service, alors que les radiologues sont déjà trop peu nombreux (Whittle, 1997 ; Whittle, 2001). Le nombre d'échographies effectuées est modéré et il varie selon les localités. En 1994, 77\% des femmes ont une échographie du premier trimestre et $80 \%$ une échographie du second trimestre au Royaume Uni (Lowther et al.1997).

Malgré le nombre limité d'échographies effectuées, c'est dans ce pays que le dépistage de la trisomie 21 par échographie est perfectionné au premier trimestre de la grossesse. A Londres, au début des années 90, un médecin se spécialise dans la mesure de la nuque fotale au premier trimestre. Il dépose un brevet sur un logiciel de calcul de risque de la trisomie 21 en fonction de cette mesure et crée un établissement privé, la Fondation pour la Médecine Fœtale, qui fournit à un prix élevé des examens aux femmes enceintes et des formations à l'échographie foetale. Il rencontre un succès certain auprès de femmes qui estiment que l'hôpital public ne leur offre pas un service suffisant en matière d'échographie fotale. En 2008, tous les services de maternité publics proposent au moins deux échographies par grossesse, au premier et au deuxième trimestre, mais seuls $30 \%$ d'entre eux proposent la mesure de la nuque fotale aux femmes enceintes (NHS, 2008). La pratique de l'échographie fœtale et la mesure de la nuque sont donc constamment plus faibles en Angleterre qu'en France, ce qui a engendré vraisemblablement moins de prescriptions de caryotypes, mais au 
prix d'inégalités géographiques et socioéconomiques. Le second type de dépistage se généralise aussi plus lentement en Angleterre qu'en France.

\section{L'organisation et le financement du dépistage biochimique}

En région parisienne, à la fin des années 80, une biochimiste qui travaille dans un hôpital universitaire et des généticiens s'intéressent aux marqueurs sériques maternels pour la trisomie 21 qui ont été mis au point en Angleterre et aux Etats-Unis en 1984. Le dosage de marqueurs au deuxième trimestre de la grossesse leur semble prometteur. Ils mettent en place une étude expérimentale pour en tester l'efficacité et l'acceptabilité dans des services d'obstétrique en région parisienne et en province de 1990 à 1993. Même si elle donne lieu à des publications scientifiques, d'autres généticiens restent sceptiques sur son intérêt, étant donné le taux d'erreur du test en terme de faux positifs. L'administration de la santé est favorable à la prise en charge par l'assurance-maladie du test de dépistage et des caryotypes qui en résulteraient, mais les ministres de la santé successifs hésitent car c'est une décision qui augmenterait le nombre d'interruptions de grossesse, ce qui peut être mal perçu par l'électorat. Le Comité Consultatif National d'Éthique est consulté à ce sujet en 1993 et donne son accord, à condition que la qualité des analyses de laboratoires soit évaluée et que les femmes enceintes soient bien informées de la procédure. Comme aucune décision politique n'est prise, certains obstétriciens continuent de proposer le test dans leur service à titre expérimental, en demandant éventuellement une participation monétaire aux femmes. Cet argent sert moins à financer les tests biochimiques, qui sont bon marché, que les coûteuses amniocentèses nécessaires par la suite. Devant l'inertie des pouvoirs publics, les responsables du Collège National des Obstétriciens et Gynécologues de France organisent en 1996 une conférence de presse à Paris pour parler du test aux journalistes de la presse féminine, en leur expliquant que les femmes demandent ces tests dans leur consultation et sont choquées de 
devoir les payer. Le Ministère charge alors M. Mattéi, qui est généticien et député membre de la majorité politique, de faire des propositions. Suite à l'audition d'une vingtaine de personnes, la plupart étant des expertes du domaine médical, scientifique et administratif, qui se révèlent favorables au financement, il recommande de faire rembourser par l'assurance maladie le test et les amniocentèses qui en découlent, pour une période de deux ans, et d'en faire ensuite une évaluation. Le gouvernement publie un arrêté en ce sens en 1997 (France, 1997a), puis un autre arrêté stipulant quel formulaire la femme enceinte doit signer pour manifester son consentement au test (France, 1997b). Après deux ans, la mesure est reconduite sans évaluation. Cet exemple montre que le financement d'une innovation peut être obtenu par une élite professionnelle et que peu d'acteurs sont intervenus dans la prise de décision (Vassy, 2006). L'usage du test se répand rapidement. L'enquête périnatale de 1998 estime qu'au moins 75\% des femmes se sont vues proposer ce test (Blondel et al., 2000). Pourquoi la généralisation est-elle si rapide, alors même que les praticiens qui suivent les grossesses travaillent dans une multitude de sites hospitaliers et libéraux éparpillés dans tout le pays ? Le remboursement par l'assurance maladie est valable sur tout le territoire national et il apporte une légitimité symbolique. De plus certains médecins interprètent à tort cette mesure économique comme une obligation de proposition systématique du dépistage ${ }^{9}$ (Grangé et Azria, 2008). D'autres prescrivent d'autant plus ce test qu'ils redoutent que des parents mécontents leur fassent un procès suite à la naissance d'un enfant malformé, si tous les moyens de dépistage n'ont pas été utilisés. La généralisation du dépistage entraîne l'augmentation du nombre de caryotypes effectués. En effet le test des marqueurs sériques, avec le seuil de risque choisi, place au moins 5\% des femmes testées dans la catégorie des risques plus élevés que la moyenne, et la plupart des femmes confrontées à cette annonce font un test diagnostique pour confirmer ou non le résultat.

\footnotetext{
${ }^{9}$ Cette obligation n'apparaitra que dans les rapports de la HAS en 2005 et 2007 (HAS, 2005 ; HAS, 2007).
} 
Au Royaume-Uni, le développement des tests de marqueurs sériques reste longtemps inégal sur le territoire, alors même que c'est dans ce pays qu'ils ont été mis au point. Un premier test a été crée en 1973 en Ecosse pour détecter le risque accru d'anencéphalie et de spina bifida, puis un autre en Angleterre en 1984 pour la trisomie 21. Dans la deuxième moitié des années 80, plusieurs équipes anglo-saxonnes trouvent d'autres marqueurs sériques corrélés avec un risque accru de trisomie 21. Des Anglais mettent au point le double test, qui mesure deux marqueurs simultanément, puis le triple test. Ils utilisent des financements pour la recherche et s'appuient sur la participation d'un petit nombre de femmes enceintes. Le premier programme de dépistage est lancé en 1990 dans deux villes du pays de Galles où il est jugé acceptable (Reynolds, 2000). Dans les années 90, le dépistage par les marqueurs sériques se généralise, mais sans couvrir toute la population car ses promoteurs sont confrontés aux contraintes budgétaires locales. En 1998, 72\% des administrations locales de la santé financent ce dépistage pour toutes les femmes enceintes, et $10 \%$ le financent avec des restrictions, en le réservant aux femmes les plus âgées (Reynolds, 2000). De nombreux praticiens critiquent cette situation et dénoncent les inégalités géographiques d'accès au dépistage. Mais il n’y a pas vraiment de débat public et le gouvernement préfère ne pas se prononcer sur ce sujet, qui pourrait susciter des controverses sur les interruptions de grossesse ou réveiller des accusations d'eugénisme (Petrogiannis et al., 2001). Des chercheurs britanniques au cours des années 90 créent de nouveaux tests des marqueurs sériques utilisables plus tôt, au premier trimestre de la grossesse, puis des logiciels qui calculent un risque unique à partir des mesures de marqueurs sériques et de la clarté nucale, en déposant parfois des brevets.

L'élite médicale britannique critique les fortes variations géographiques du dépistage biochimique, en terme de groupes de femmes concernées, de tests utilisés et de seuils de risques retenus (Wald et al. 1998 ; Whittle, 2001). Elle demande l'intervention de l'Etat pour savoir quels sont les tests qu'il faut financer (Reynolds, 2000). En 2000, le Ministre de la 
Santé annonce la création d'un réseau de coordinateurs régionaux et locaux pour former les praticiens et harmoniser les pratiques du dépistage de la trisomie 21. Ce réseau, constitué principalement de sages-femmes en milieu hospitalier, est placé sous l'égide du Comité National de Dépistage (National Screening Committee), qui a été créé en 1996 pour évaluer tous les programmes de dépistage sanitaire. En 2001, le Ministre de la santé annonce un plan de modernisation des services de maternité, qui comprend l'obligation de proposer au moins une forme de dépistage de la trisomie 21 à toutes les femmes enceintes. Ce plan est à réaliser avant trois ans. La même année, le Comité National de Dépistage annonce un programme national de dépistage de la trisomie 21 . Il recommande aux services de maternité de proposer un dépistage par les marqueurs sériques qui fournisse un taux de détection au moins égal à $60 \%$ pour un taux de faux positifs de $5 \%$ maximum. La décision de fixer des taux standards est habile car elle évite de se prononcer pour un test en particulier, et donc de trancher entre différents intérêts économiques (Kerr, 2004). Par la suite le Comité National a révisé ses recommandations en s'appuyant sur des experts, et a demandé un meilleur taux de détection des fœtus trisomiques et un moindre taux de faux positifs, à atteindre en quelques années. Pour inciter les responsables hospitaliers à financer les dépistages recommandés, les coordinateurs locaux leur rappellent régulièrement que des parents mécontents pourraient leur faire des procès s'ils n'ont pas reçu le service désigné comme le meilleur. L'offre de dépistage s'harmonise donc lentement au cours des années 2000.

Dans les deux pays, les dépistages biochimiques se sont développés sur des initiatives locales de chercheurs et praticiens, et les gouvernements sont intervenus tardivement sous la pression d'une élite professionnelle pour assurer l'égalité d'accès. Mais les gouvernements ne sont pas intervenus à la même époque, ni dans le même contexte, et leurs interventions n'ont pas le même effet. L'intervention des pouvoirs publics en Angleterre est plus tardive et elle s'inscrit dans un système de financement des soins décentralisé. A la fin de la décennie 90 , le 
dépistage par marqueurs sériques est plus développé en France qu'en Angleterre, alors même que les tests ont été créés au Royaume-Uni.

\section{Impact sur le nombre de naissances d'enfants trisomiques}

Quel est l'impact de ces politiques de dépistage et de diagnostic sur le nombre de naissances d'enfants porteurs de trisomie 21 ? Dans les deux pays, la décennie 1990-2000 se traduit par une baisse importante du nombre de ces naissances. Les données épidémiologiques manquent dans ce domaine, surtout au niveau national, car les estimations sont souvent faites à partir de registres locaux, dans lesquels sont enregistrées les naissances avec des malformations. De plus ces chiffres sont à interpréter avec précaution car les recensions de ces naissances ne sont pas toujours exhaustives. D'après l'étude de Morris et al. (2002), le nombre de fœtus trisomiques 21 détectés avant la naissance en Angleterre et au Pays de Galle serait passé de $30 \%$ à $62 \%$ entre 1989 et 2002 . La majorité des cas détectés (92\%) aurait donné lieu à un avortement médicalisé. Le nombre de naissances d'enfants avec trisomie 21 serait passé de 750 en 1989 à 570 en 2002, soit une diminution de presque un tiers (Morris et al, 2002). En France, on ne dispose des données que pour certaines régions où il y a des registres. Il faut faire des extrapolations au niveau national. Le nombre de naissances d'enfants trisomiques 21 serait passé de 800-900 en 1990 à 500-600 en 2001 (Goujard, 2004), soit une diminution d'un tiers également. Mais une autre estimation avance une baisse plus importante sur la même période (Flori et al., 2006).

\section{Conclusion}

En Angleterre comme en France, les logiques d'acteurs à l'origine de la généralisation des tests de dépistage et diagnostic de la trisomie 21 sont semblables. Des chercheurs et des praticiens du système de santé public créent ou importent des tests. Les initiatives de ces élites 
scientifiques et cliniques trouvent un premier public de femmes enceintes et mettent les pouvoirs publics devant un problème délicat : quel encadrement règlementaire et financier trouver pour ces techniques qui suscitent de l'intérêt, mais aussi des critiques ? Cela a posé aussi des défis aux praticiens qui ont été amenés à les intégrer à leur pratique courante : comment s'y former, comment en parler aux femmes enceintes et éventuellement comment financer les équipements nécessaires ? Enfin ces initiatives ont ouvert de nouvelles possibilités aux femmes enceintes, mais aussi de nouvelles responsabilités et inquiétudes. Dans les deux pays, les pouvoirs publics ont progressivement financé la réalisation des tests, mais sans donner les moyens humains supplémentaires pour assurer une information de qualité aux femmes enceintes. Aujourd'hui cette information est souvent encore insuffisante, entraînant des critiques de médecins, de chercheurs en sciences humaines et sociales et d'associations représentant les usagers, dont certains dénoncent des pratiques d'imposition de techniques médicales.

Dans les deux pays, ce sont les mêmes tests qui ont été adoptés, mais pas aux mêmes dates, ni avec la même fréquence. Notre étude montre l'importance des caractéristiques institutionnelles des systèmes de santé pour expliquer ces différences dans l'adoption des innovations biomédicales. La France se distingue par un plus grand usage des tests. Le mode de financement des soins y est public, comme en Angleterre, mais centralisé. Un petit nombre d'acteurs a été impliqué dans les décisions de financement de ces nouveaux actes médicaux par l'Assurance Maladie, et ces décisions ont eu immédiatement une portée nationale, ce qui a accéléré la diffusion. Le système anglais, en revanche, a un mode de financement décentralisé, qui repose sur les négociations d'un grand nombre d'acteurs au niveau local (Appleby \& Gregory, 2008). Ils doivent établir des priorités dans le financement de diverses activités biomédicales, ce qui s'est fait souvent au détriment du dépistage prénatal. Une autre caractéristique institutionnelle importante est la présence d'un secteur libéral en France, avec 
un paiement à l'acte par l'Assurance Maladie. Dans le cas de l'échographie, ce sont ces praticiens qui ont choisi d'utiliser la nouvelle technique de dépistage et d'investir dans des équipements, alors que le secteur public était plus contraint en France et en Angleterre. Enfin le suivi de chaque grossesse repose en France sur un plus grand nombre de professionnels qu'en Angleterre, avec des intervenants libéraux et hospitaliers qui ne sont pas toujours coordonnés. D’où la mise en place au cours des années 1990 d'un dépistage « en cascade » ou séquentiel, où la femme enceinte est testée à plusieurs reprises au cours de sa grossesse pour la trisomie 21 (échographie du premier trimestre, marqueurs sériques du deuxième trimestre, échographie du deuxièmetrimestre), ce qui entraîne un cumul des faux-positifs de chaque test et la réalisation de nombreux tests diagnostics pour confirmer ou non ces résultats (Ville, 2005; Boog 2003, Rozenberg, 2005). Le système français a donc permis de diminuer les inégalités géographiques et socio-économiques dans l'accès aux tests pendant la décennie 1990. Mais il a entraîné la multiplication des tests, ce qui a un coût économique pour l'Assurance-Maladie et un coût psychologique pour les femmes désignées comme à haut risque et qui perdent éventuellement un foetus sain dans la procédure.

A l'avenir, dans la décennie 2010, le problème du nombre trop élevé de caryotypes fœtauxen France devrait s'atténuer. Depuis janvier 2011 l'assurance maladie finance un dépistage intégré, qui consiste à calculer une seule fois le risque de trisomie 21 en début de grossesse, à partir de la mesure de la nuque fotale et d'un test des marqueurs sériques faits d'une manière rapprochée au premier trimestre. De plus la mise en place de réseaux de périnatalité devrait améliorer la coordination entre les professionnels qui s'occupent d'une même grossesse et peut-être améliorer l'information des femmes enceintes.

Le système de santé anglais a davantage restreint l'accès aux tests de dépistage et de diagnostic prénatals. Il y a donc moins de coûts économiques et psychologiques. Mais il s’est 
créé des inégalités géographiques d'accès : les femmes se sont vu proposer des tests plus ou moins nombreux et performants selon l'établissement de santé qu'elles fréquentaient. Il s'est aussi créé des inégalités socioéconomiques, les personnes les plus aisées et les mieux informées allant chercher des test supplémentaires, par exemple des échographies avec mesure de la clarté nucale, dans le secteur privé. Ce n'est qu'au cours de la décennie 2000, que les pouvoirs publics ont pris des mesures pour harmoniser les pratiques. Les responsables hospitaliers ont été incités à financer des programmes de dépistage prénatal performants. Le passage au dépistage prénatal du premier trimestre y est quasiment achevé, mais il n'est pas sûr que les problèmes d'information des femmes enceintes y soient résolus.

Davantage de recherches sont nécessaires pour savoir si la diffusion des innovations est plus rapide en France qu'en Angleterre pour d'autres techniques biomédicales que ces tests prénatals. La comparaison avec d'autres techniques de dépistage qui se sont largement diffusées en France, comme la mammographie pour le cancer du sein ou le test de dépistage néonatal de la mucoviscidose, laisse penser que le système de santé français serait particulièrement favorable à la généralisation des tests de dépistage, même quand leur extension n'a pas fait toute la preuve de son utilité sur le plan de la santé publique (Boissonnat, 2004 ; Vailly, 2004).

\section{Remerciements :}

Cette recherche a été en partie financée grâce au programme «Sciences biomédicales, santé et société» du CNRS-INSERM-MIRE en 2004, et au programme ANR Sciences et société en 2010.

\section{Bibliographie :}


Agence de la Biomédecine, 2007, Rapport Annuel : Bilan des activités de l'Agence, SaintDenis.

ANAES, 1998, L'échographie obstétricale au cours de la grossesse en l'absence de facteur de risque, Rapport de l'Agence Nationale d'Accréditation et d'Evaluation des Etablissements de Santé.

Appleby, J \& Gregory, S. (2008). NHS spending - local variations in priorities: an update.

London, The King's Fund.

Baszanger I., Gaudillière J.P., Löwy I. 2000, Avant-Propos, Sciences Sociales et Santé, 18, 4, 4-8.

Bateman-Novaes S. 1998, La bioéthique comme objet sociologique, Cahiers Internationaux de Sociologie, 104, 5-32.

Blondel B, Du Mazaubrun C, Breart G. 1995, Enquête nationale périnatale 1995. Rapport de fin d'étude.Paris, INSERM, Unité 149.

Blondel B., J. Norton, C. du Mazaubrun, G. Breart, 2000, Enquête périnatale de 1998, Paris, Rapport de l’Unité 149 Inserm.

Boissonnat, V. (2004). Fausses évidences et vrais dilemmes en santé publique, Le dépistage radiologique du cancer du sein. Santé Publique, 16, 1, 9-20.

Bourret P., Huard, P. 1990, Création, appropriation, recomposition. Processus innovants dans la production du diagnostic prénatal, Sciences sociales et santé, 8, 4, 57-90. 
Carles E., Eydoux P. 2002 Cytogénétique prénatale , bilan 1999 pour l'Association des Cytogénéticiens de Langue Française, Médecine Fotale et Echographie en Gynécologie, 50, $5-8$.

Carricaburu D. 2005 De la gestion technique du risque à celle du travail : l'accouchement en hôpital public, Sociologie du Travail, 47, 2, Pages 245-262.

Champenois-Rousseau B. 2003 Éthique et moralité ordinaire dans la pratique du diagnostic prénatal, Thèse en socioéconomie de l'innovation sous la direction de M. Akrich, Paris, Ecole des Mines.

Chitty L. 1995, Ultrasound screening for fetal abnormalities, Prenatal diagnosis, 15, 12411257.

Clarke A.J. 1997, Prenatal genetic screening. Paradigms and perspectives, in P. Harper \& A.J. Clarke, Genetics, society and clinical practice, BIOS Scientific Publishers, 119-140.

De Vigan, C., Vodovar, V., Goujard, J., Garel, M., Vayssière, C., \& Goffinet, F. 2002,Mothers' knowledge of screening for trisomy 21 in 1999, A survey in Paris maternity units. European Journal of Obstetrics and Gynecology, 104, 1, 14-20.

David, D., \& Gosme-Seguret, S. (Eds.). 1996,Le diagnostic prénatal, aspects psychologiques. Paris,ESF.

Dommergues M. et Bessis R., 2003, Rapport sur l'échographie foetale, Syndicat national de l'union des échographistes. Accessible sur www.snude.org.

Farrant, W. (1985). Who's for amniocentesis? The politics of prenatal screening. In H. Homans (Ed.), The sexual politics of reproduction. Aldershot, Gower, 96-122 
Favre R. (2004) Dépistage de la trisomie 21. Un consentement éclairé a-t-il été réalisé ? Mémoire de DEA de Sciences Biologiques et Médicales et d'Ethique. Université de Strasbourg.

Favre, R. 2007, En quoi le niveau de connaissance médicale et la position des médecins respectent-ils ou non le consentement des patientes dans le cadre du dépistage de la Trisomie 21 ? Thèse de droit, santé et éthique médicale de l'université Paris V.

Fellous M. 1988 La révolution échographique, Sociologie du travail, n² 2, 301-322

Flori M, Goffette J, 2006 Dépistage du risque de la trisomie 21 par les marqueurs sériques. Réflexions pratiques et éthiques. La revue du praticien, 20, 734, 2-5.

France, 1997a, Arrêté du 23 janvier 1997 modifiant l'arrêté du 3 avril 1985 fixant la Nomenclature des actes de biologie médicale, Journal Officiel de la République Française.

France, 1997b, Arrêté du 30 septembre 1997 relatif au consentement de la femme enceinte à la réalisation des analyses mentionnées à l'article R-162-16-1 du code de la santé publique, Journal Officiel de la République Française.

Gaudillière J-P, 2006, La médecine et les sciences. XIXe XXe siècles. Paris, La Découverte.

Gaudillière J-P, 2001, Bettering Babies. Down's Syndrome, Heredity and Public Health in Post-War France and Britain, in I. Löwy \& J. Krige, Images of Disease. Office for Official Publications of the European Communities.

Gekas J. et al. (1999) Informed consent to serum screening for Down Syndrome.Are women given adequate information? Prenatal Diagnosis, 19, 1-7. 
Goujard J. 2004. La mesure de la clarté nucale et le dosage des marqueurs sériques commencent-ils à modifier l'incidence de la trisomie 21 en France ? Gynécologie Obstétrique et Fertilité, 06, 32 (6), 496-501.

Grangé G, Azria E, 2008, Entre proposition systématique et obligation d'information, quelles libertés face au dépistage de la trisomie 21 ?, Journal de gynécologie obstétrique et biologie de la reproduction, $37,6,535-537$

Green, J. (1994). Serum screening for Down's syndrome, Experience of obstetricians in England and Wales. British Medical Journal, 309, 769-772.

Green J. \& Statham H., 1996, Psychosocial aspects of prenatal screening and diagnosis, in T. Marteau \& M. Richards, The troubled helix. Social and psychological implications of the new human genetics, Cambridge University Press, 140-163.

Green J., Hewison J., Bekker H, Bryant L, Cuckle H, 2004, Psychosocial aspects of genetic screening of pregnant women and newborns, a systematic review, Health Technology Assessment, 8, 33, 1-124.

Hagard S. \& Carter, F.A. 1976 Preventing the birth of infants with Down's syndrome, a costbenefit analysis, British Medical Journal, 1,753-756.

HAS 2005 Comment mieux informer les femmes enceintes? Recommandations professionnelles, Rapport de la Haute Autorité de Santé, St Denis.

HAS 2007 Evaluation des stratégies de dépistage de la trisomie 21, Rapport de la Haute Autorité de Santé, St Denis.

Heyman B., \& Henriksen M. (2001) Risk, age and pregnancy. A case study of prenatal genetic screening and testing, Basingstoke, Palgrave. 
Hopkins M, 2006, The hidden research system, the evolution of cytogenetic testing in the National Health Service, Science as Culture, 15(3), 253-276.

ICHBD, 2007, Annual Report 2007, International Clearinghouse for Birth Defects Surveillance \& Research, http,//www.icbdsr.org.

Jacques B. 2007, Sociologie de l'accouchement, Paris, PUF.

Julian-Reynier C. et Bourret P. 2006, Techniques bio-médicales et pratiques sélectives ; choix individuels, choix collectifs ? in Jean Gayon et Daniel Jacobi, L'éternel retour de l'eugénisme, Paris, PUF, 61-92.

Kerr A., 2004, Genetics \& Society. A sociology of disease. London, Routledge.

Khoshnood B, De Vigan C., Blondel B., Lhomme A., Vodovar V., Garel M., Goffinet F. 2006, Women's interpretation of an abnormal result on measurement of fetal nuchal translucency and maternal serum screening for prenatal testing of Down syndrome, Ultrasound Obstet. Gynecol., 28, 242-248.

Lascoumes P., Le Galès P. 2007, Sociologie de l'action publique, Paris, Armand Colin.

Nicolaides KH, Snijders RJM, Gosdem CM, Berry C, Campbell S. 1992 Ultrasonographically detectable markers of fetal chromosomal abnormalities.Lancet,340, 704-7.

Lowther G, Whittle M.1997 Prenatal diagnosis in the United Kingdom- an overview,European Journal of Human Genetics 5, 1, 84-9.

Marteau, T. (1995) Towards informed decisions about prenatal testing, a review, Prenatal Diagnosis, 15, 1215-1226. 
Marteau T.M., Slack J. Kidd J. \& Shaw R.W. (1992) Presenting a routine screening test in antenatal care, practice observed. Public Health, 106, 2, 131-41.

Mattéi J.F. (2000) Le diagnostic prénatal Paris, Flammarion.

Musselin C. 2005 Sociologie de l'action organisée et analyse des politiques publiques, deux approches pour un même objet ? Revue française de Sciences politiques, 55, 1, 51-72.

NHS Fetal Anomaly Screening Programme, 2008, Screening for Down's syndrome.

UK NSC Policy recommendations 2007-2010, Model of Best Practice, Londres, Ministère de la Santé.

Nisand, I. 2001, Vers la réification de l'être humain ? Le Monde, 12.07.2001.

Oakley A. 2003, Essays on Women, Medicine and Health, Edinburgh University Press.

Petrogiannis K., Tymstra T., Jallinoja P., Ettore E., 2001, Review of policy, law and ethics, in E. Ettore, Before birth. Understanding prenatal screening, Aldershot, Ashgate, 38-52.

Pinell, 2004a, Diagnostic prénatal, in D. Lecourt, Dictionnaire de la pensée médicale, Paris, PUF.

Pinell 2004b, Génétique médicale, in D. Lecourt, Dictionnaire de la pensée médicale, Paris, PUF.

Reid, M. (1991) The diffusion of four prenatal screening tests across Europe. London, King's Fund Centre.

Reynolds T, 2000, Down's syndrome screening, a controversial test, with more controversy to come !, Journal of Clinical Pathology, 53, 893-898. 
Robinson, J. 1997, False alarm, but real harm ? British Journal of Midwifery, 5, 12, 777.

Rozenberg P. (2005) Dépistage de la trisomie 21 par échographie, Gynécologie, obstétrique et fertilité, 33, 7, 526-532.

Schwartz Cowan R., 2008, Heredity \& Hope. The case for genetic screening, Harvard University Press.

Seror V., Costet N, Aymé S, 2001, Participation in maternal serum marker screening for Down Syndrome: Contribution of the information delivered to the decision making process, Community Genetics, 4, 158-172.

Seror V. 2003 Organisation et régulation de l'activité du diagnostic prénatal. Point de vue économique, in M. Dommergues, S. Aymé, P. Janiaud et V. Seror, Diagnostic prénatal, pratiques et enjeux. Paris, Editions INSERM, 506-518.

Smith D., Shaw R \& Marteau T, 1994a Lack of knowledge in health professionals: a barrier to providing information to patients, Quality in Health Care, 3, 75-78.

Smith D, Shaw R \& Marteau T, 1994b, Informed consent to undergo serum screening for Down's syndrome : the gap between policy and practice, British Medical Journal 309, 776.

Smith-Bindman R., Chu Ph., Baccheti, P., Waters J., Mutton D., Alberman, E., 2003, Prenatal screening for Down syndrome in England and Wales and population-based birth outcomes, American Journal of Obstetrics \& Gynecology, 189, 4, 980-985.

Statham H., Green J. 1993 Serum screening for Down's syndrome : some women's experiences, British Medical Journal 307, 174-6. 
Steffen, M. (1996). Genèse et conduite des politiques sociales. In S. Aymé, J. C. Henrard, A.

Colvez, J. F. Ravaud, O. Sabouraud, \& A. Triomphe (Eds.), Handicap et vieillissement:

Politiques publiques et pratiques sociales. Paris: INSERM.

Steffen M. (2004) La comparaison internationale comme révélateur des apprentissages institutionnels ; l'exemple de la lutte contre le sida, Revue Internationale de Politique Comparée, 11, 3, 389-411.

Stocking, B. (1991). Factors affecting the diffusion of three kinds of innovative medical technology in European Community Countries and Sweden. London: King's Fund Centre.

Sureau Cl. et Henrion R., 2005, Rapport du comité national technique de l'échographie de dépistage prénatal pour le Ministre de la Santé, Paris.

Vailly, J. (2004). Une politique de santé ‘a priori’: Le dépistage néonatal de la mucoviscidose en Bretagne. Sciences Sociales et Santé, 22(4), 35-60.

Vassy C. (2006) From a genetic innovation to mass health programmes: The diffusion of Down's Syndrome prenatal screening and diagnostic techniques in France, Social Science \& Medicine, 63, 8, pp. 2041-2051.

Vassy C. et Keller R., 2010, Cross National Qualitative Health Research, in Bourgeault I.L., DeVries R., Dingwall R. (eds), Sage Handbook of Qualitative Methods in Health Research, London, Sage, 621-638.

Ville Y. 2005, Le dépistage prénatal doit être expliqué aux femmes enceintes mais aussi à leurs médecins, Blog du Collectif Interassociatif Autour de la Naissance, Accessible sur : http://www.ciane.info/article-2001187.html. 
Whittle, M., 1997, Ultrasonographic soft markers of fetal chromosomal defects. Detecting them may do more harm than good, British Medical Journal, 314, 918.

Whittle M. 2001, Down's syndrome screening: where to now? British Journal of Obstetrics \& Gynecology, 108, 544-561.

Williams Cl. Alderson P. Farsides B, 2002, Is nondirectiveness possible within the context of antenatal screening and testing? Social Science \& Medicine, 54, 339-347. 